Supporting information for

\title{
"Inhibitory activity of insulin on A $\beta$ aggregation is restricted due to binding selectivity and specificity to polymorphic A $\beta$ states"
}

\begin{abstract}
Michal Baram ${ }^{1,2}$ and Yifat Miller ${ }^{1,2, *}$
${ }^{1}$ Department of Chemistry Ben-Gurion University of the Negev, Be'er Sheva 84105, Israel

${ }^{2}$ The Ilse Katz Institute for Nanoscale Science \& Technology Ben-Gurion University of the Negev, Be'er Sheva 84105, Israel
\end{abstract}

Corresponding author:

Yifat Miller: ymiller@bgu.ac.il 


\section{Material and Methods}

\section{Constructions of insulin-A $\beta$ fibril-like oligomers}

Three polymorphic models of $A \beta$ fibril-like hexamers were constructed based on ssNMR (Figure 1). Two structural models are "U-shape" A $\beta$ fibril-like hexamers: models $\mathrm{A}$ and $\mathrm{B}, 1,2$ and one structural model is "S-shape" $\mathrm{A} \beta$ fibril-like hexamer model C. ${ }^{3}$ The insulin molecule that has been applied in the current study was taken from the crystal structure, pdb id code: $1 \mathrm{GUJ}{ }^{4}$

A total of ten different insulin-A $\beta$ fibril-like hexamers were constructed. The binding of the insulin molecule with each one of the three polymorphic $A \beta$ fibril-like hexamers (model A, B and C) take into account the recognition motif for amyloids. ${ }^{5}$ The recognition motif of insulin B chain - residues F24-F25-Y26 - was bound to the $\beta$ strand domain in $\mathrm{A} \beta$ : residues $\mathrm{K} 16-\mathrm{L} 17-\mathrm{V} 18-\mathrm{F} 19-\mathrm{F} 20$. The insulin molecule was interacted with each one of the three polymorphic $A \beta$ fibril-like hexamers - models $A$, $\mathrm{B}$ and $\mathrm{C}$ - in two locations. In the first location the insulin was bound across the fibril to one of the central monomers within the hexamer (Figure 2). In this location, two orientations were taken into account in the recognition motif. In the second location, the insulin molecule was bound along the fibril axis of $A \beta$ (Figure 5). Similar to the first location, also in this location two orientations were taken into account in the recognition motif.

Four models were constructed for insulin that was bound across $A \beta$ fibril: models A1 and A2 (Figure S5), and B1 and B2 (Figure S6). Due to steric effects along the "Sshape" $A \beta$ fibril of model $C$, the insulin molecule was not able to bound across the fibril (Figure S4). Six models were constructed for insulin that bound along A $\beta$ fibril axis: models A3 and A4 (Figure S16), models B3 and B4 (Figure S17), and models C1 and C2 (Figure S18).

\section{Determining the conformational energies and populations for the simulated models of $A \beta$ fibril-like oligomers and insulin- $A \beta$ fibril-like oligomers}

To obtain the relative structural stability of the variant models, the trajectories of the last $5 \mathrm{~ns}$ were first extracted from the explicit MD simulation excluding water molecules. The solvation energies of all systems were calculated using the Generalized Born Method with Molecular Volume (GBMV). ${ }^{6,7}$ In the GBMV calculations, the dielectric constant of water was set to 80.0. The hydrophobic solvent-accessible surface 
area (SASA) term factor was set to $0.00592 \mathrm{kcal} / \mathrm{mol} \cdot \AA^{2}$. Each variant is minimized 1000 cycles and the conformation energy is evaluated by grid-based GBMV. The minimization does not change the conformations of each variant, but only relaxed the local geometries due to thermal fluctuation which occurred during the MD simulations. A total of 5,000 conformations (500 conformations for each of the 10 examined conformers) were used to construct the free energy landscape of the conformers and to evaluate the conformer probabilities by using Monte Carlo (MC) simulations. In the first step, one conformation of conformer $i$ and one conformation of conformer $j$ were randomly selected. Then, the Boltzmann factor was computed as e-(Ej-Ei)/kT, where $\mathrm{Ei}$ and $\mathrm{Ej}$ are the conformational energies evaluated using the GBMV calculations for conformations $\mathrm{i}$ and $\mathrm{j}$, respectively, $\mathrm{k}$ is the Boltzmann constant and $\mathrm{T}$ is the absolute temperature ( $298 \mathrm{~K}$ used here). If the value of the Boltzmann factor was larger than the random number, then the move from conformation $i$ to conformation $j$ was allowed. After 1 million steps, the conformations 'visited' for each conformer were counted. Finally, the relative probability of model $n$ was evaluated as $\mathrm{Pn}=\mathrm{Nn} / \mathrm{Ntotal}$, where $\mathrm{Pn}$ is the population of model $\mathrm{n}, \mathrm{Nn}$ is the total number of conformations visited for model $\mathrm{n}$, and Ntotal is the total steps. The advantages of using MC simulations to estimate conformer probability lie in their good numerical stability and the control that they allow of transition probabilities among several conformers .

Using a total number of models and 5,000 conformations (500 for each model) generated from the MD simulations, we estimated the overall stability and populations for each conformer based on the MD simulations, with the energy landscape being computed with GBMV for these models.

\section{Determining hydrophobic, electrostatic and $\pi-\pi$ interactions}

The hydrophobic interactions between two residues were estimated by measurements of the distances between $\mathrm{C} \alpha$ atoms of two residues. The cutoff distance for hydrophobic interactions is $10 \AA .^{8}$ The electrostatic interactions between two residues were estimated by the distance measurements between $\mathrm{O}$ atom of the acidic residue and the $\mathrm{N}$ atom of the base residue. The cutoff distance for electrostatic interactions is $4 \AA .{ }^{9}$ The cutoff distance between $C$ atom of two aromatic residue that estimated the $\pi-\pi$ interactions is $7 \AA .^{10}$ 


\section{Structural analysis details}

We determine the structural stabilities of the models by following the changes in the average percentage of hydrogen bonds between $\beta$-strands, with the hydrogen bond cutoff being set to $2.5 \AA$. This examination was performed by following the root-mean square deviations (RMSDs) and root-mean square fluctuations (RMSFs). The RMSF were calculated by averaging the RMSF values of backbone and sidechain of each residue along the sequence of $A \beta$ for all studied models. To estimate the secondary structure of the $A \beta$ fibril models and insulin, the hydrogen bond estimation algorithm Define Secondary Structure of Proteins (DSSP) was applied in the last 5 ns of the simulation for each studied model. 


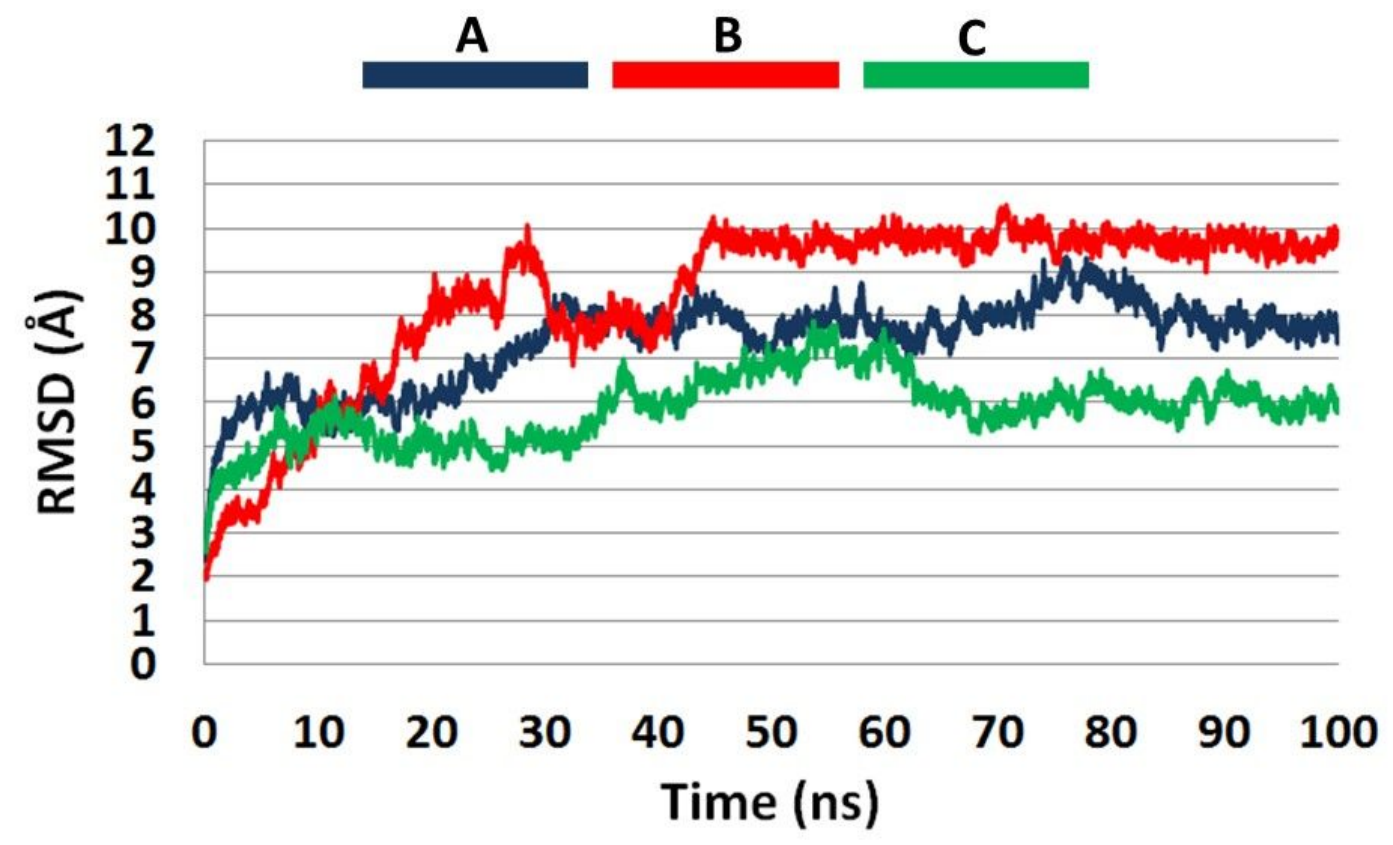

Figure S1: RMSDs of A $\beta$ fibril-like hexamers of models A, B and C, as a function of time. 


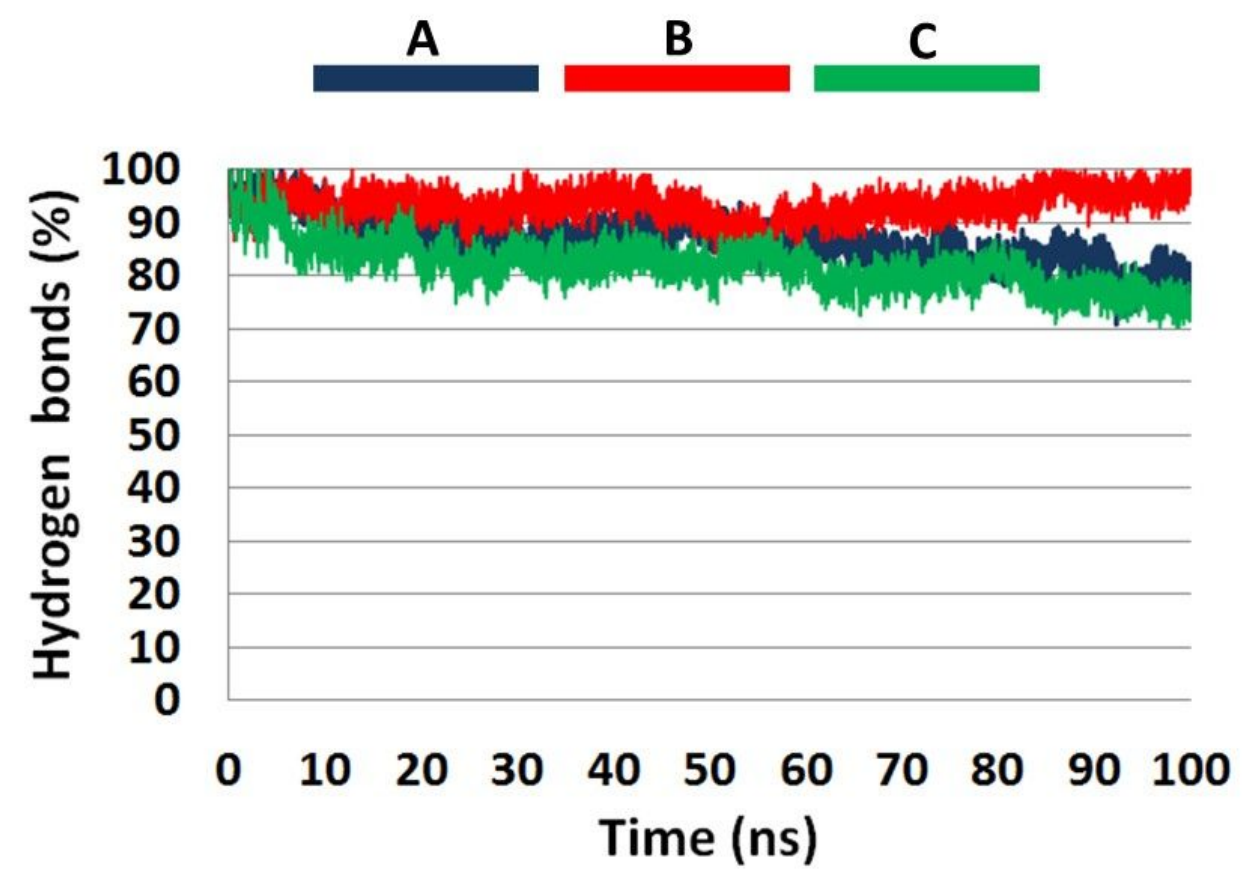

Figure S2: The fraction of the number of hydrogen bonds (in percentage) between all $\beta$-strands in $A \beta$ fibril-like hexamers of models $A, B$ and $C$ as a function of time. 


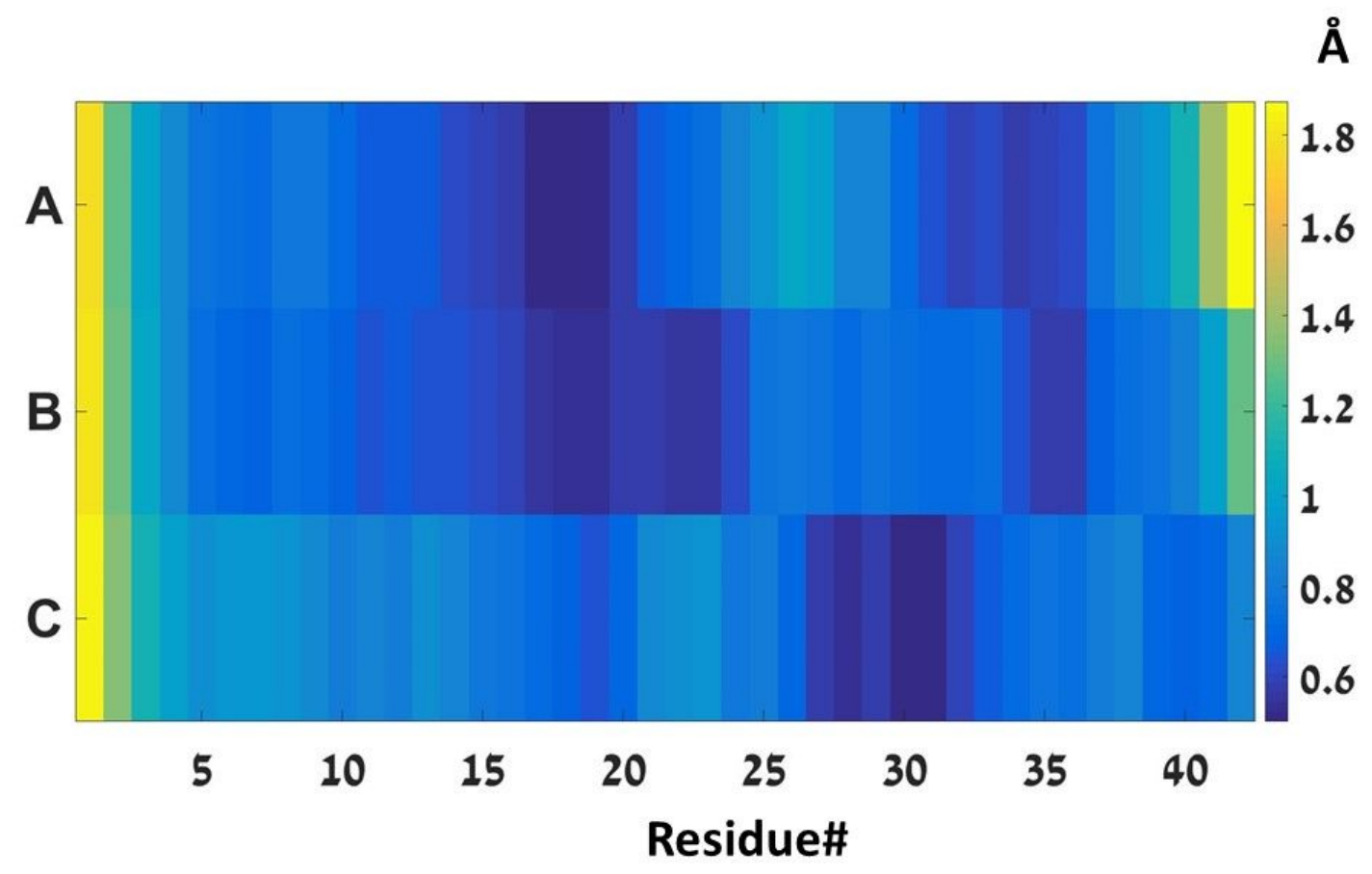

Figure S3: RMSFs of each residue along $A \beta$ sequence for $A \beta$ fibril-like hexamers of models A, B and C. 


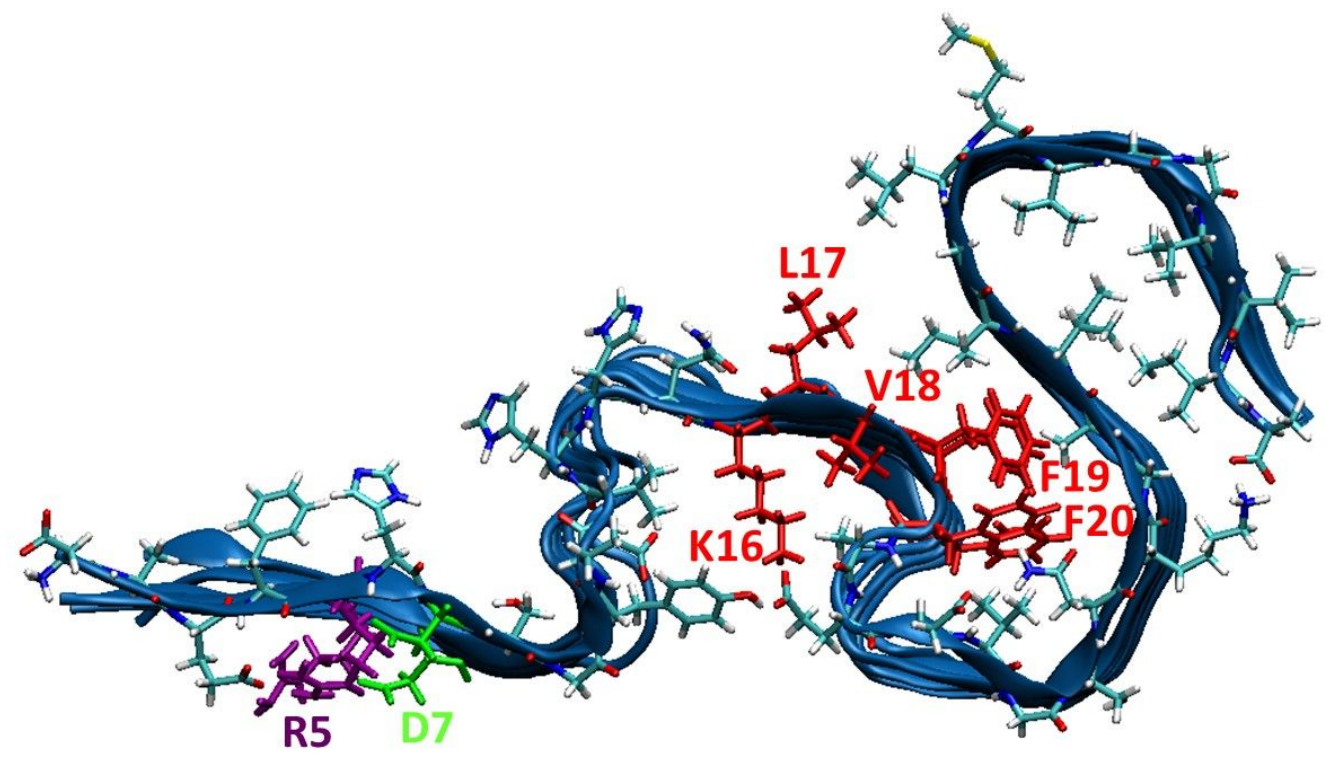

Figure S4: The structure of "S-shape" fibril-like structure, based on ssNMR. ${ }^{3}$ The recognition motif (residues in red color, are seen only for one monomer within the hexamer) is buried, thus does not exposed to the binding with insulin across the fibril. The inter- and intra-molecular interactions between R5 and D7 stabilize the "S-shape" fibril. 


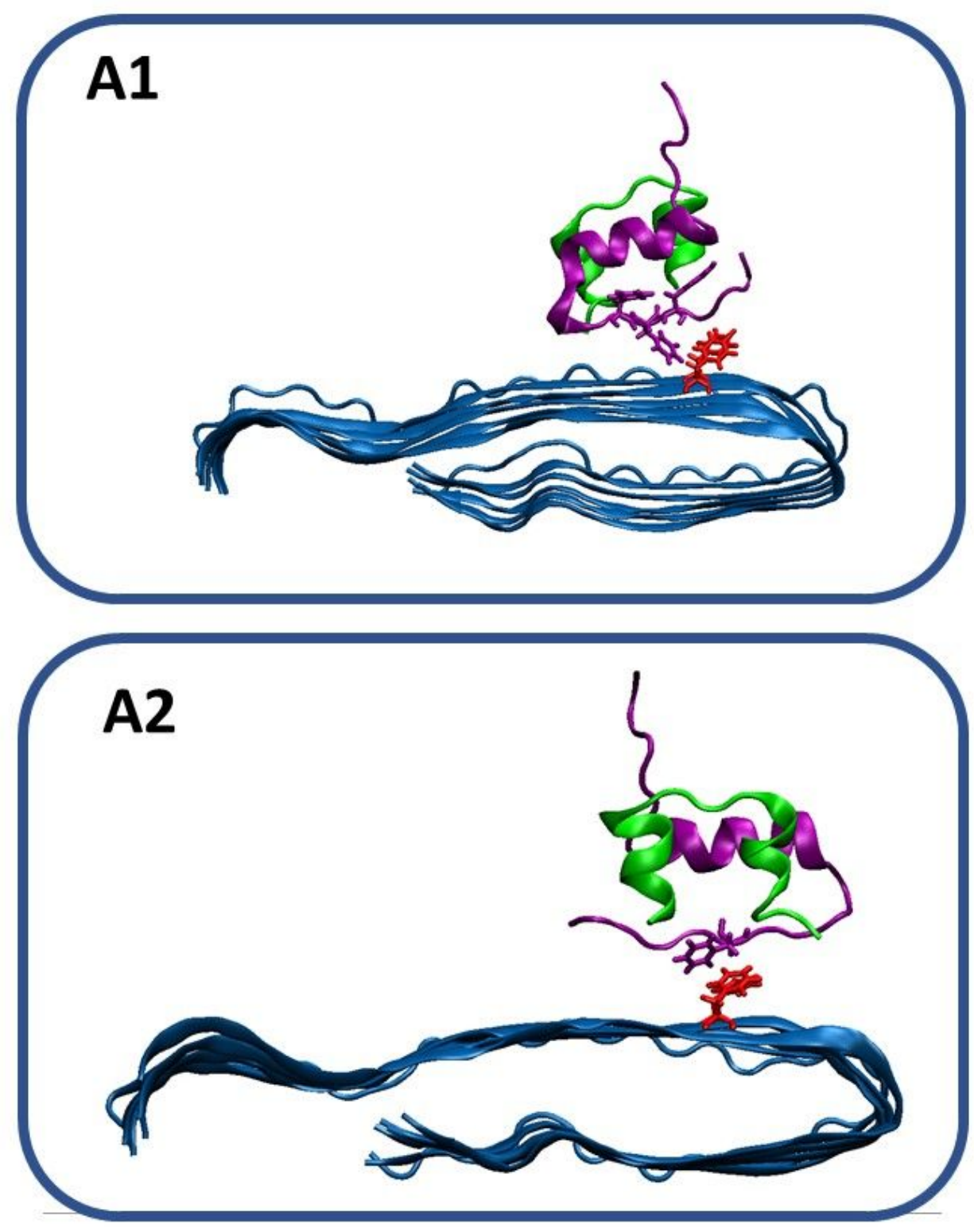

Figure S5: Initial structures of insulin binding to "U-shape" $A \beta_{1-42}$ fibril-like hexamers in two orientations across the fibril, as seen schematically in Figure 2. The "U-shape" $\mathrm{A} \beta_{1-42}$ fibril-like hexamers are based on ssNMR. ${ }^{2}$ 


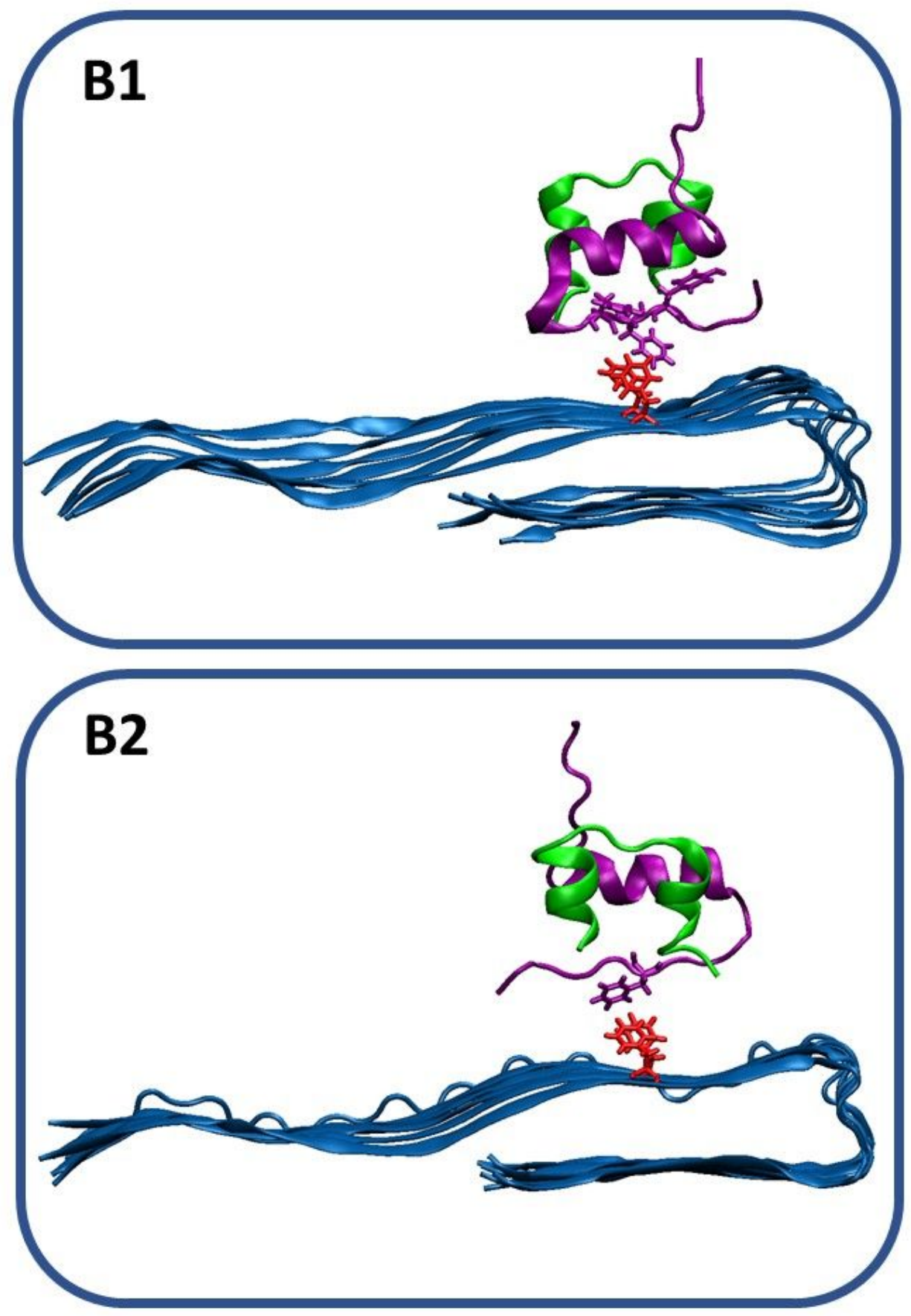

Figure S6: Initial structures of insulin binding to "U-shape" $\mathrm{A} \beta_{1-42}$ fibril-like hexamers in two orientations across the fibril, as seen schematically in Figure 2. The "U-shape" $\mathrm{A} \beta_{1-42}$ fibril-like hexamers are based on ssNMR. ${ }^{1}$ 


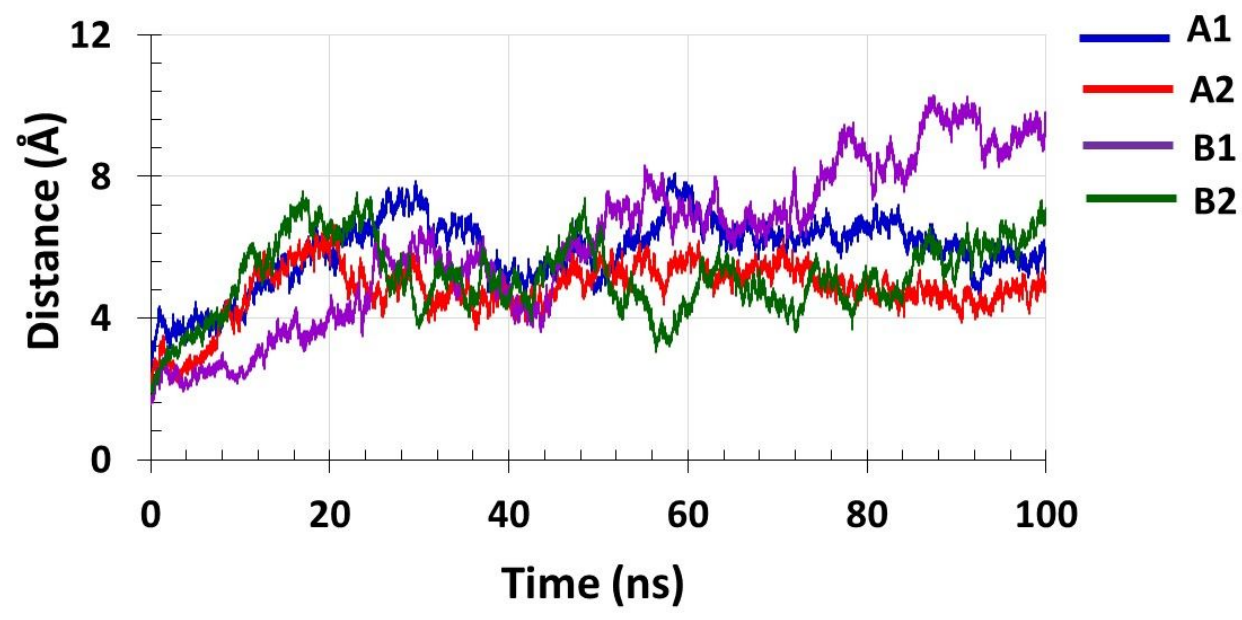

Figure S7: RMSDs of insulin-A $\beta$ fibril-like hexamers of models A1, A2, B1 and B2, as a function of time. 


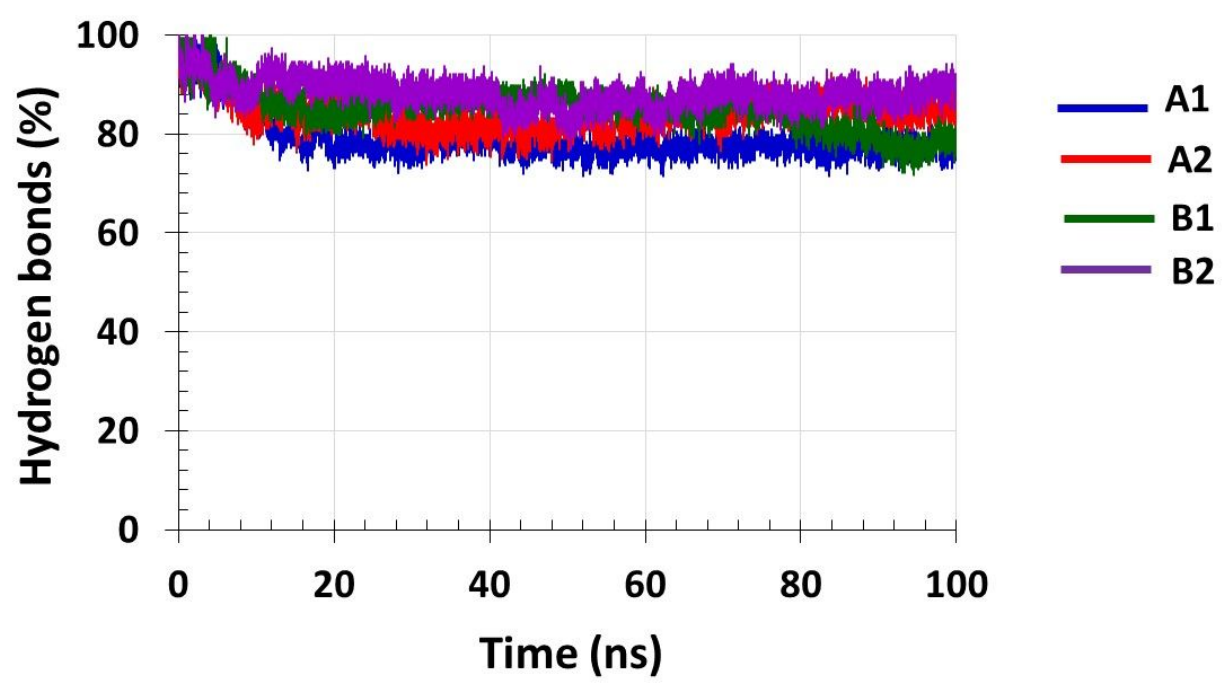

Figure S8: The fraction of the number of hydrogen bonds (in percentage) between all $\beta$-strands in A $\beta$ fibril-like hexamers in models A1, A2, B1 and B2 as a function of time. 


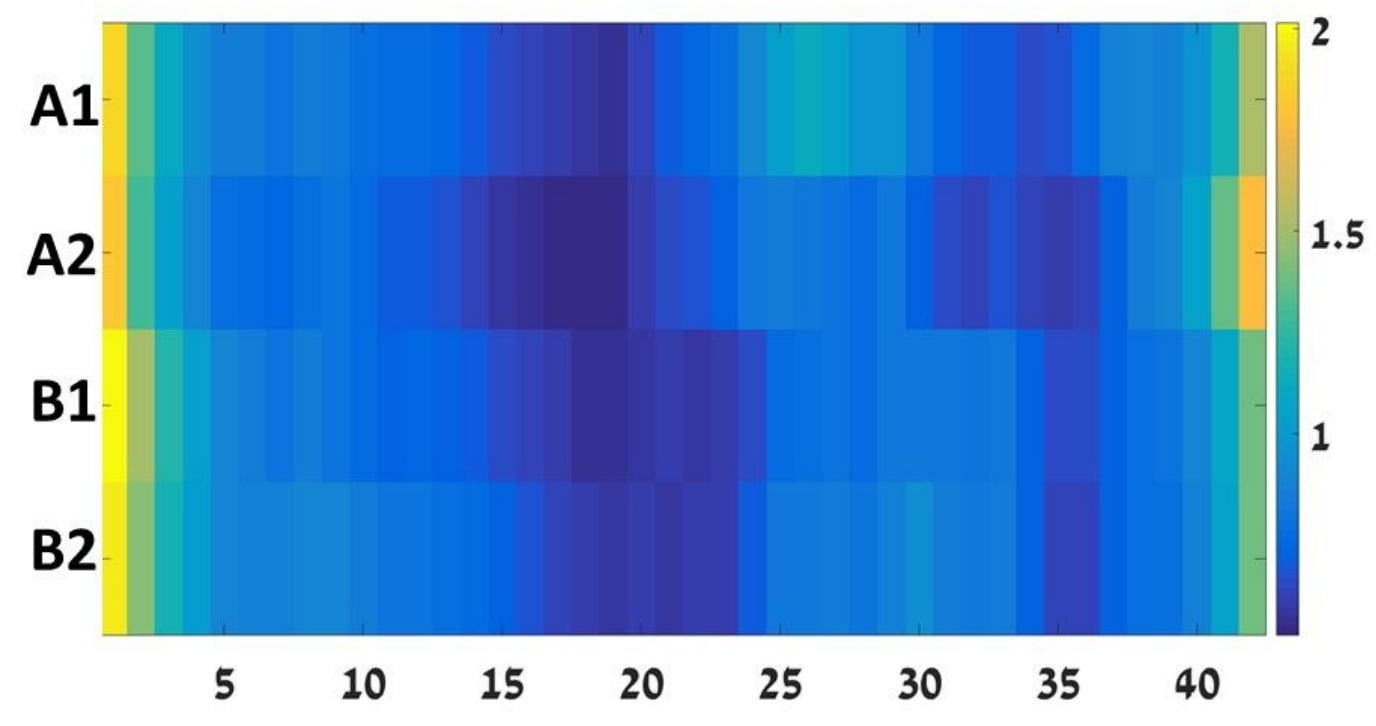

Figure S9: RMSFs of each residue along $A \beta$ sequence for $A \beta$ fibril-like hexamers in models A1, A2, B1 and B2. 


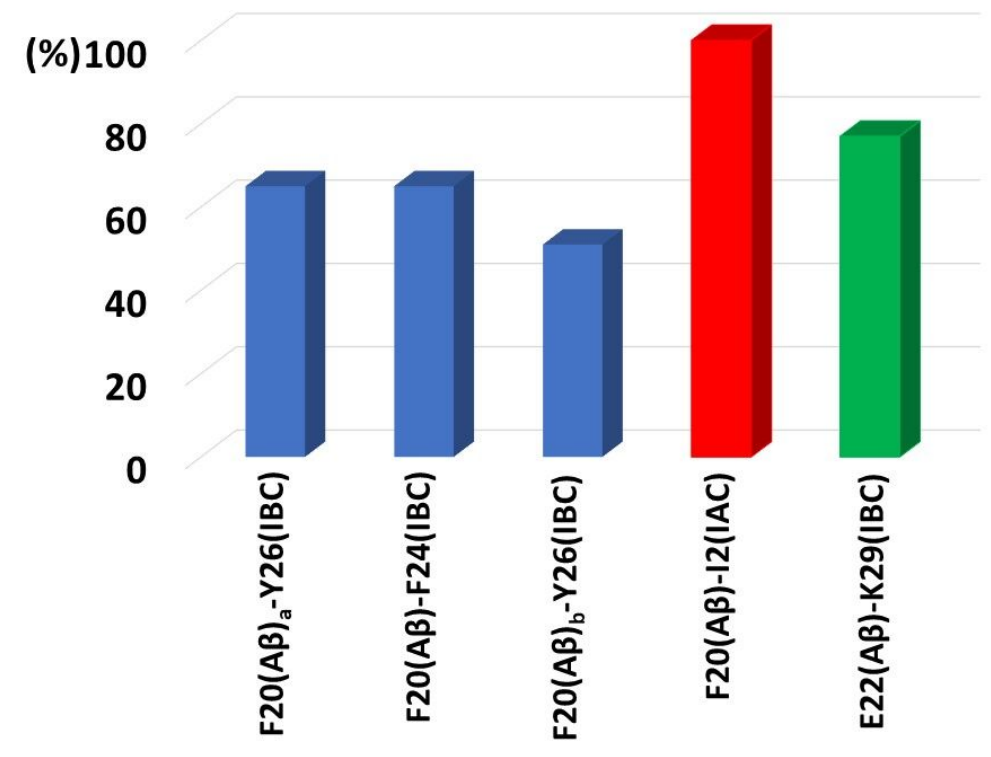

Figure S10: The total percentage of the interactions between residues in insulin and residues in $A \beta$ within $A \beta$ fibril-like hexamers for model $A 1$. Interactions between residues in insulin and two different $A \beta$ are termed as a and $b$. The $\pi$ - $\pi$ interactions are colored in blue, hydrophobic interactions are colored in red and electrostatic interactions are colored in green. Only interactions that displayed $>50 \%$ of the time of simulations are presented. 


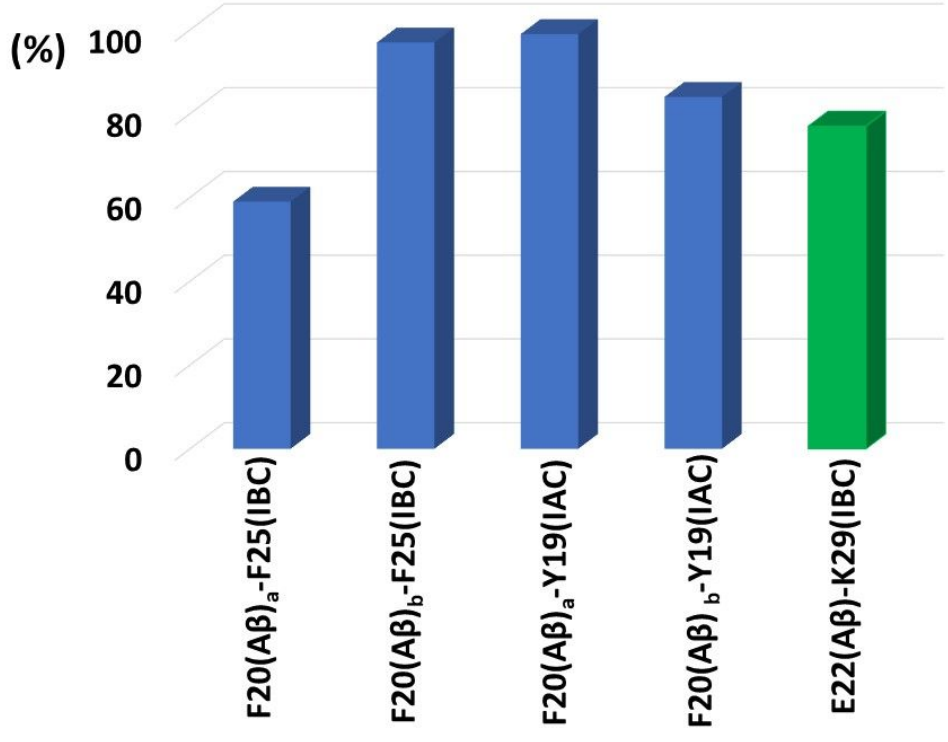

Figure S11: The total percentage of the interactions between residues in insulin and residues in $A \beta$ within $A \beta$ fibril-like hexamers for model $B 1$. Interactions between residues in insulin and two different $A \beta$ are termed as a and $b$. The $\pi-\pi$ interactions are colored in blue and electrostatic interactions are colored in green. Only interactions that displayed $>50 \%$ of the time of simulations are presented. 
(\%)

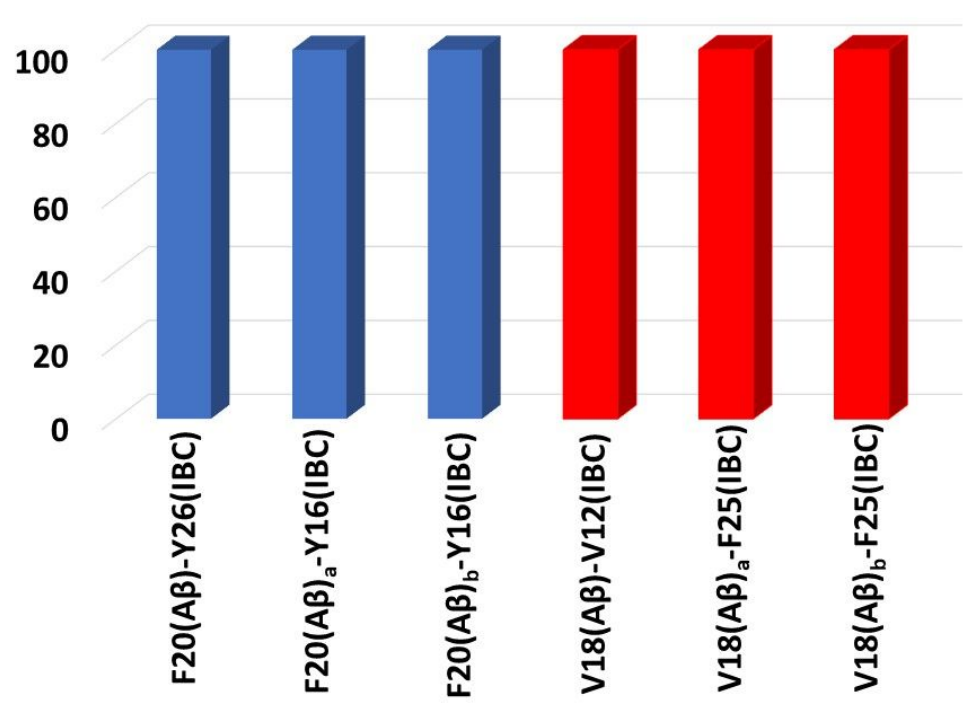

Figure S12: The total percentage of the interactions between residues in insulin and residues in $A \beta$ within $A \beta$ fibril-like hexamers for model $A 2$. Interactions between residues in insulin and two different $A \beta$ are termed as a and $b$. The $\pi$ - $\pi$ interactions are colored in blue and hydrophobic interactions are colored in red. Only interactions that displayed $>50 \%$ of the time of simulations are presented. 
(\%)

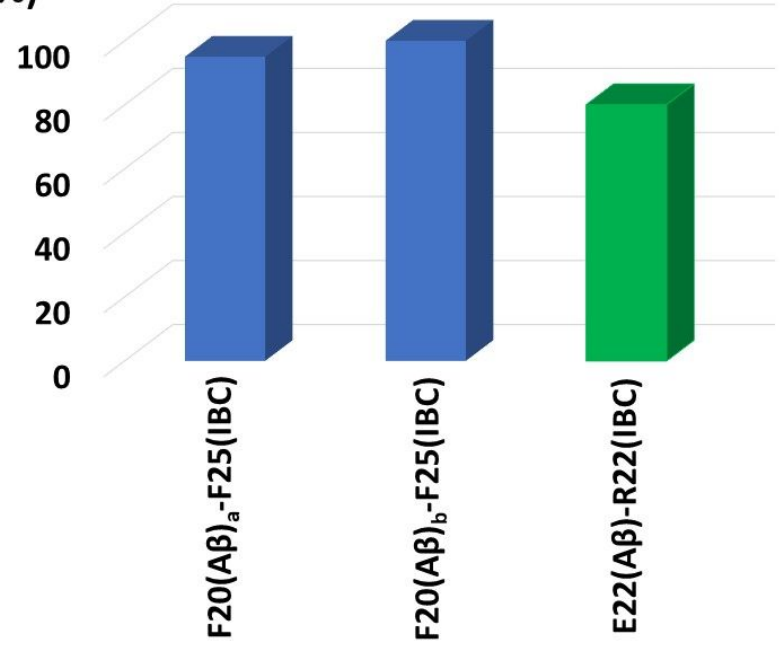

Figure 13: The total percentage of the interactions between residues in insulin and residues in $A \beta$ within $A \beta$ fibril-like hexamers for model B2. Interactions between residues in insulin and two different $A \beta$ are termed as a and $b$. The $\pi$ - $\pi$ interactions are colored in blue and electrostatic interactions are colored in green. Only interactions that displayed $>50 \%$ of the time of simulations are presented. 


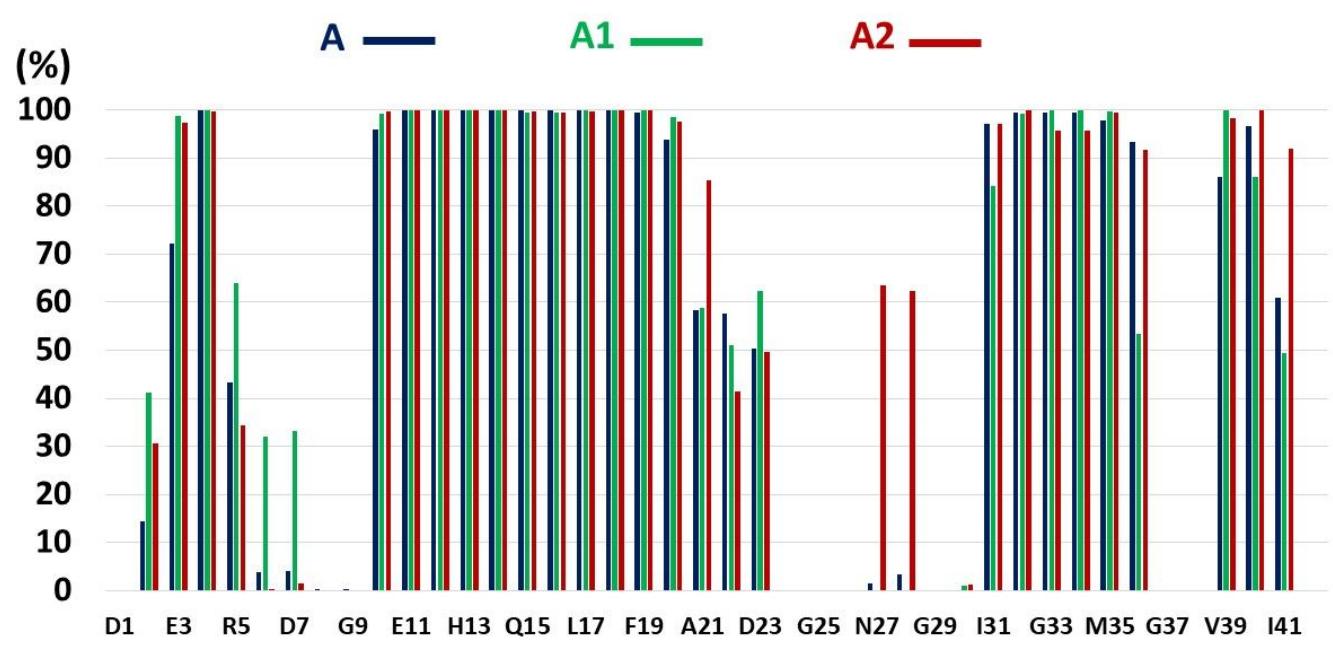

Figure S14: DSSP analysis for models A, A1 and A2 calculated from the last 5 ns. 


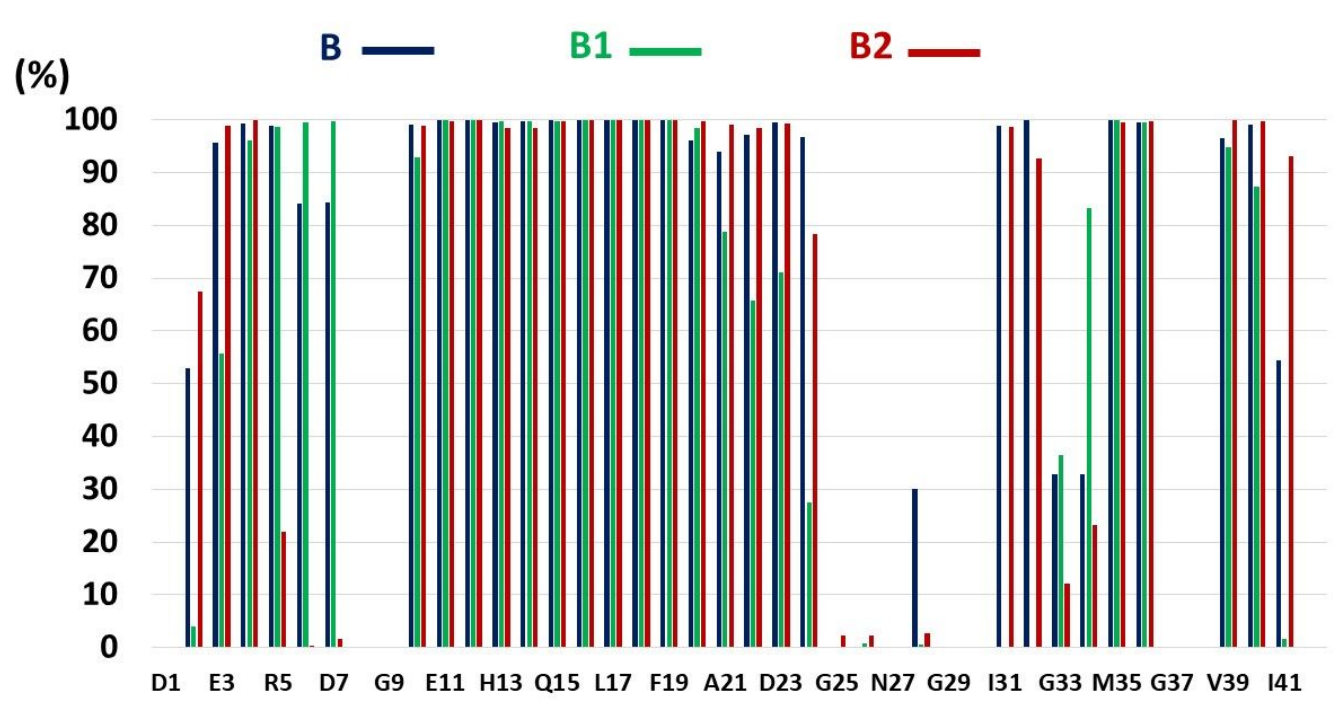

Figure S15: DSSP analysis for models B, B1 and B2 calculated from the last 5 ns. 


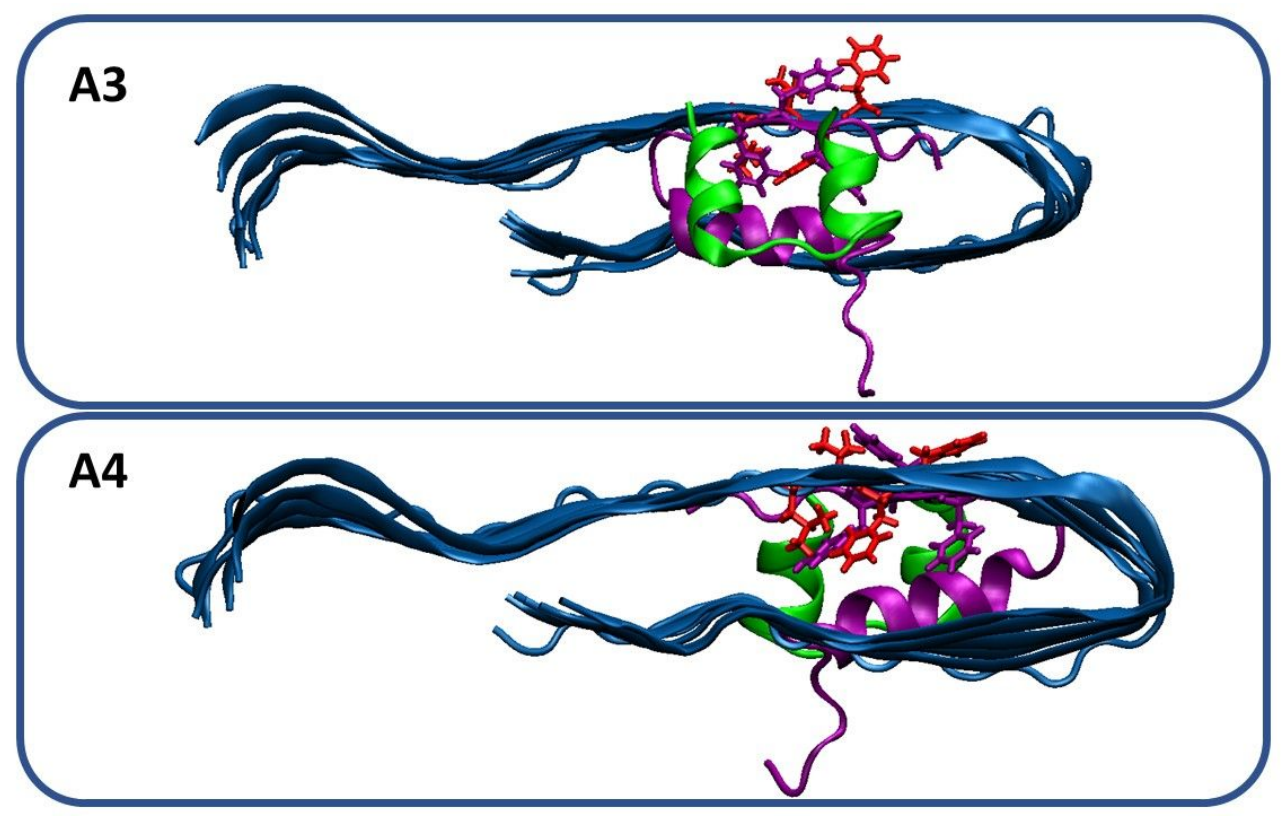

Figure S16: Initial structures of insulin binding to "U-shape" $A \beta_{1-42}$ fibril-like hexamers in two orientations along the fibril, as seen schematically in Figure 5. The "U-shape" $\mathrm{A} \beta_{1-42}$ fibril-like hexamers are based on ssNMR. ${ }^{2}$ 


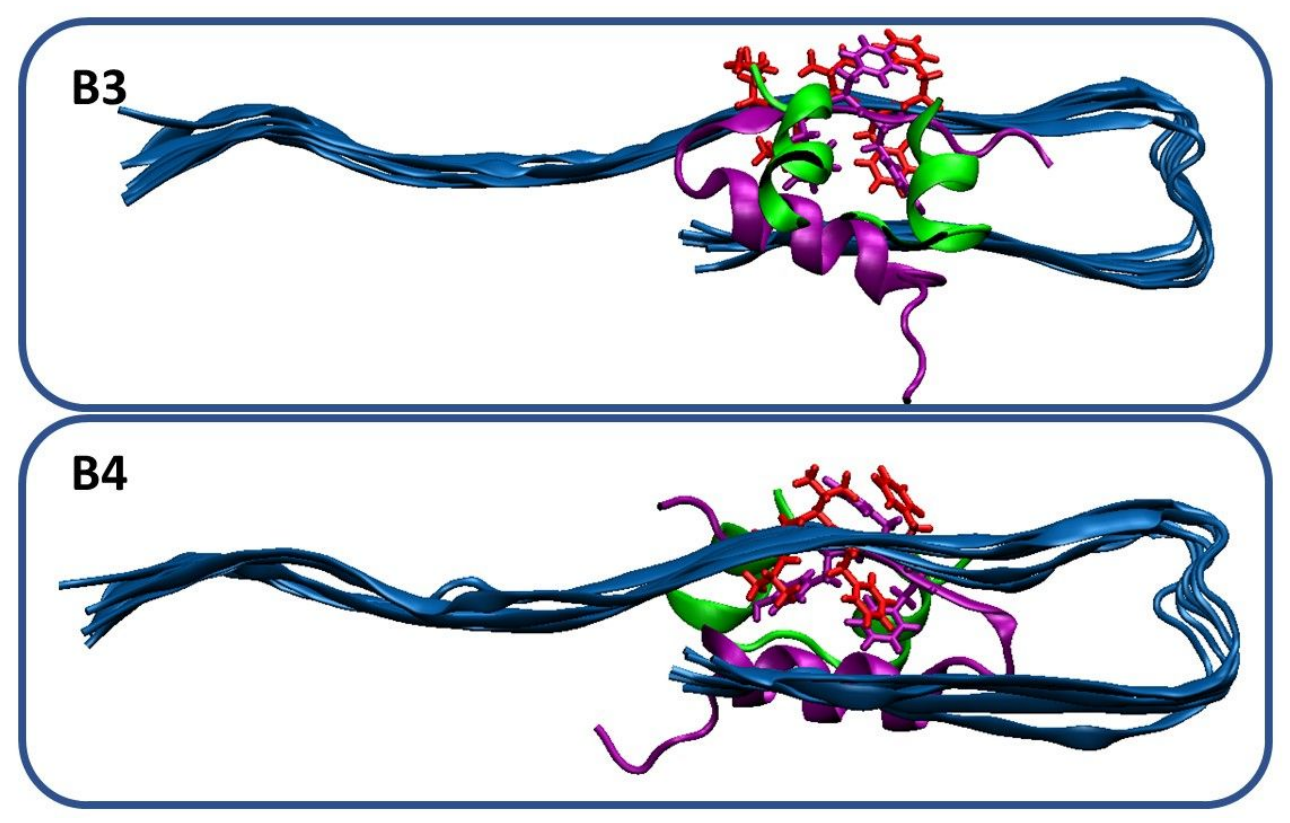

Figure S17: Initial structures of insulin binding to "U-shape" $A \beta_{1-42}$ fibril-like hexamers in two orientations along the fibril, as seen schematically in Figure 5. The "U-shape" $A \beta_{1-42}$ fibril-like hexamers are based on ssNMR. ${ }^{1}$ 


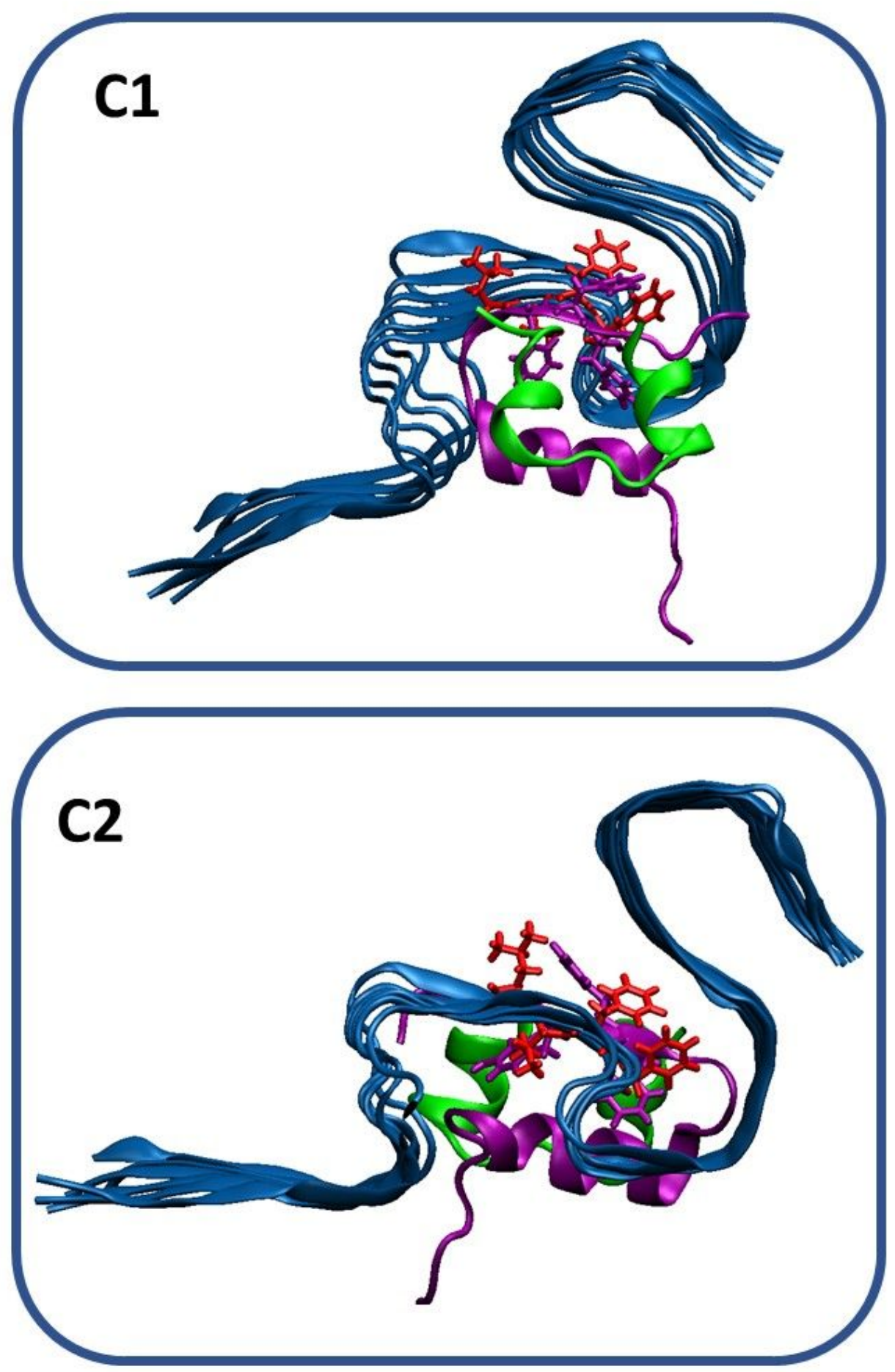

Figure S18: Initial structures of insulin binding to "S-shape" $A \beta_{1-42}$ fibril-like hexamers in two orientations along the fibril, as seen schematically in Figure 5. The "S-shape" $\mathrm{A} \beta_{1-42}$ fibril-like hexamers are based on ssNMR. ${ }^{3}$ 


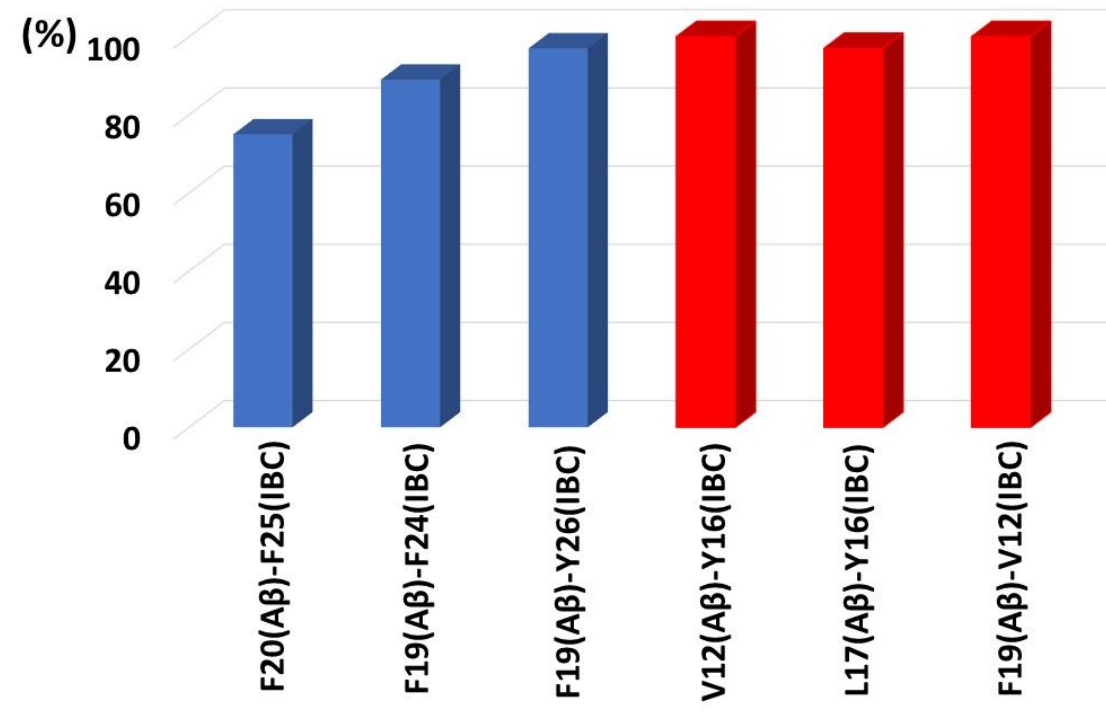

Figure S19: The total percentage of the interactions between residues in insulin and residues in $A \beta$ within $A \beta$ fibril-like hexamers for model $A 3$. Interactions between residues in insulin and two different $A \beta$ are termed as a and $b$. The $\pi-\pi$ interactions are colored in blue and hydrophobic interactions are colored in red. Only interactions that displayed $>50 \%$ of the time of simulations are presented. 

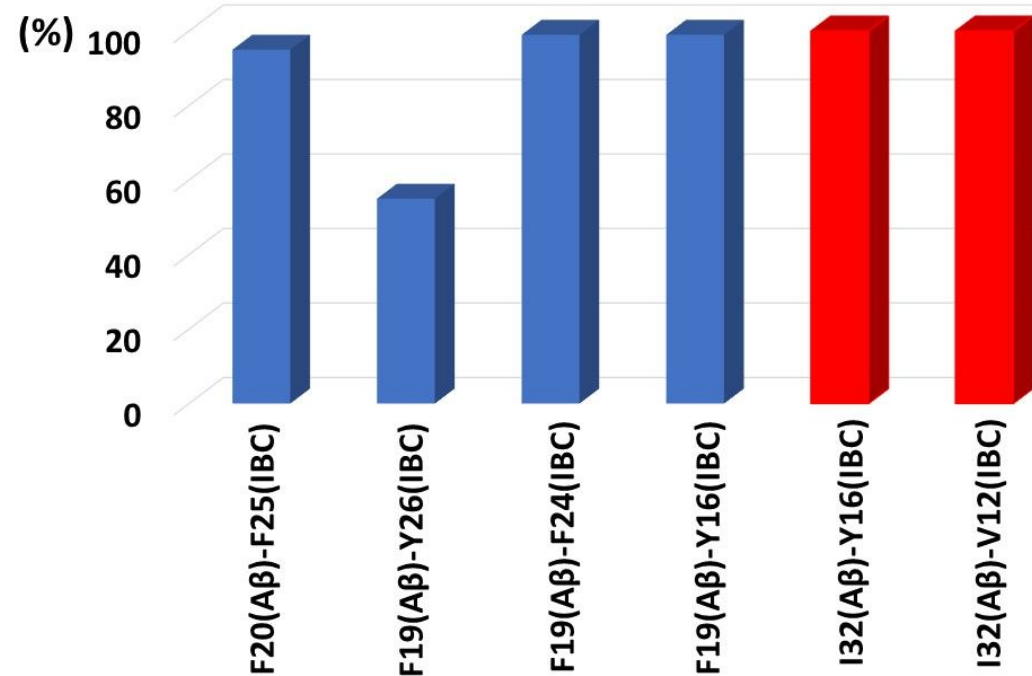

Figure S20: The total percentage of the interactions between residues in insulin and residues in $A \beta$ within $A \beta$ fibril-like hexamers for model $A 4$. Interactions between residues in insulin and two different $A \beta$ are termed as a and $b$. The $\pi-\pi$ interactions are colored in blue and hydrophobic interactions are colored in red. Only interactions that displayed $>50 \%$ of the time of simulations are presented. 
(\%) 100

80

60

40

20

0
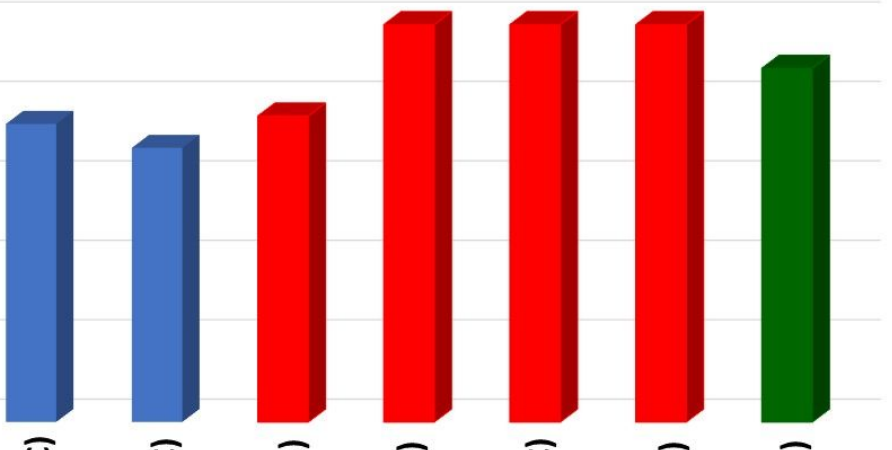

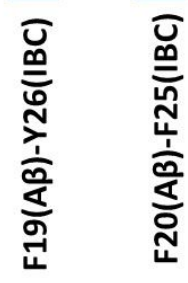

$\overline{0}$
$\frac{0}{4}$
$\frac{1}{1}$
$\frac{1}{0}$
$\frac{1}{4}$

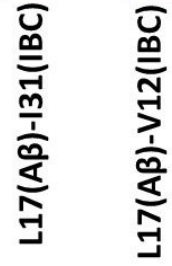

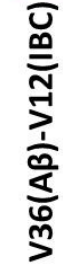

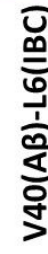

ত্̣

Figure S21: The total percentage of the interactions between residues in insulin and residues in $A \beta$ within $A \beta$ fibril-like hexamers for model $B$ 4. Interactions between residues in insulin and two different $A \beta$ are termed as a and $b$. The $\pi-\pi$ interactions are colored in blue, hydrophobic interactions are colored in red and electrostatic interactions are colored in green. Only interactions that displayed $>50 \%$ of the time of simulations are presented. 
(\%)

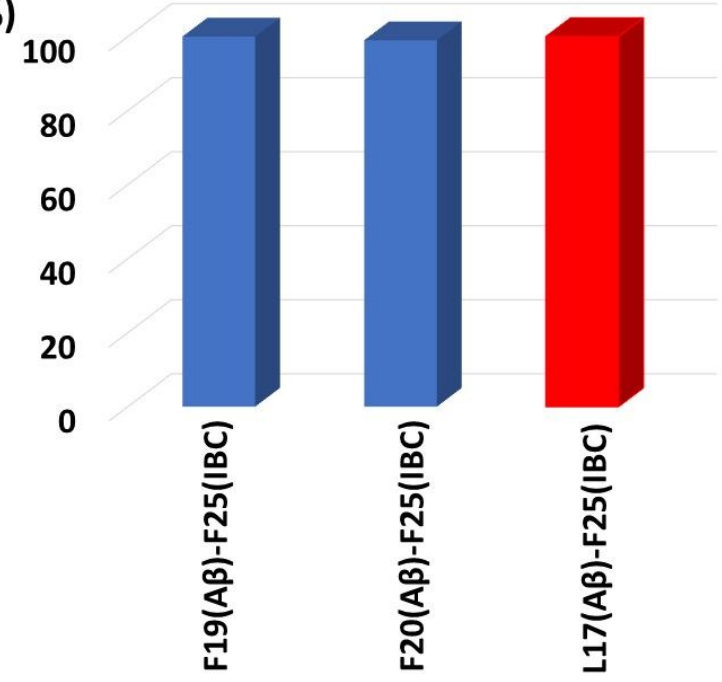

Figure S22: The total percentage of the interactions between residues in insulin and residues in $A \beta$ within $A \beta$ fibril-like hexamers for model $C 2$. Interactions between residues in insulin and two different $A \beta$ are termed as a and $b$. The $\pi-\pi$ interactions are colored in blue and hydrophobic interactions are colored in red. Only interactions that displayed $>50 \%$ of the time of simulations are presented. 


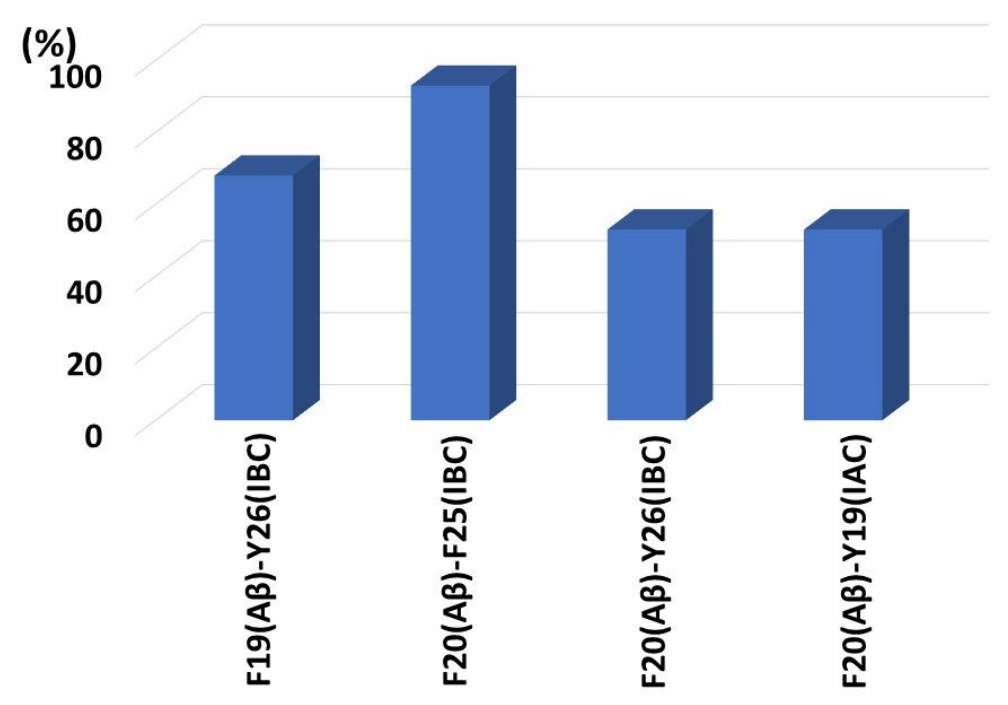

Figure S23: The total percentage of the interactions between residues in insulin and residues in $A \beta$ within $A \beta$ fibril-like hexamers for model $B 3$. Interactions between residues in insulin and two different $A \beta$ are termed as a and $b$. The $\pi-\pi$ interactions are colored in blue. Only interactions that displayed $>50 \%$ of the time of simulations are presented. 
(\%)

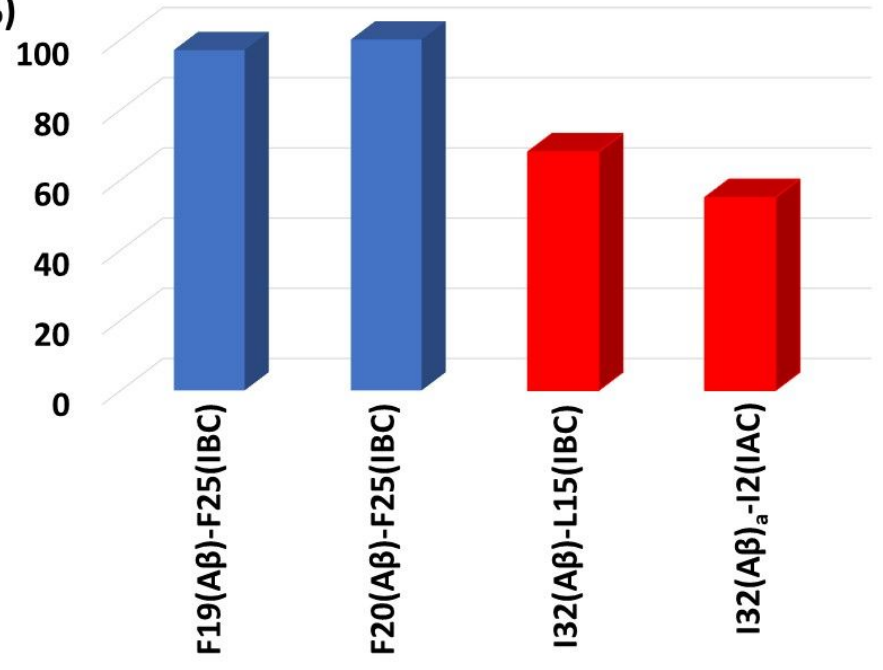

Figure S24: The total percentage of the interactions between residues in insulin and residues in $A \beta$ within $A \beta$ fibril-like hexamers for model $\mathrm{C} 1$. Interactions between residues in insulin and two different $A \beta$ are termed as a and $b$. The $\pi$ - $\pi$ interactions are colored in blue and hydrophobic interactions are colored in red. Only interactions that displayed $>50 \%$ of the time of simulations are presented. 


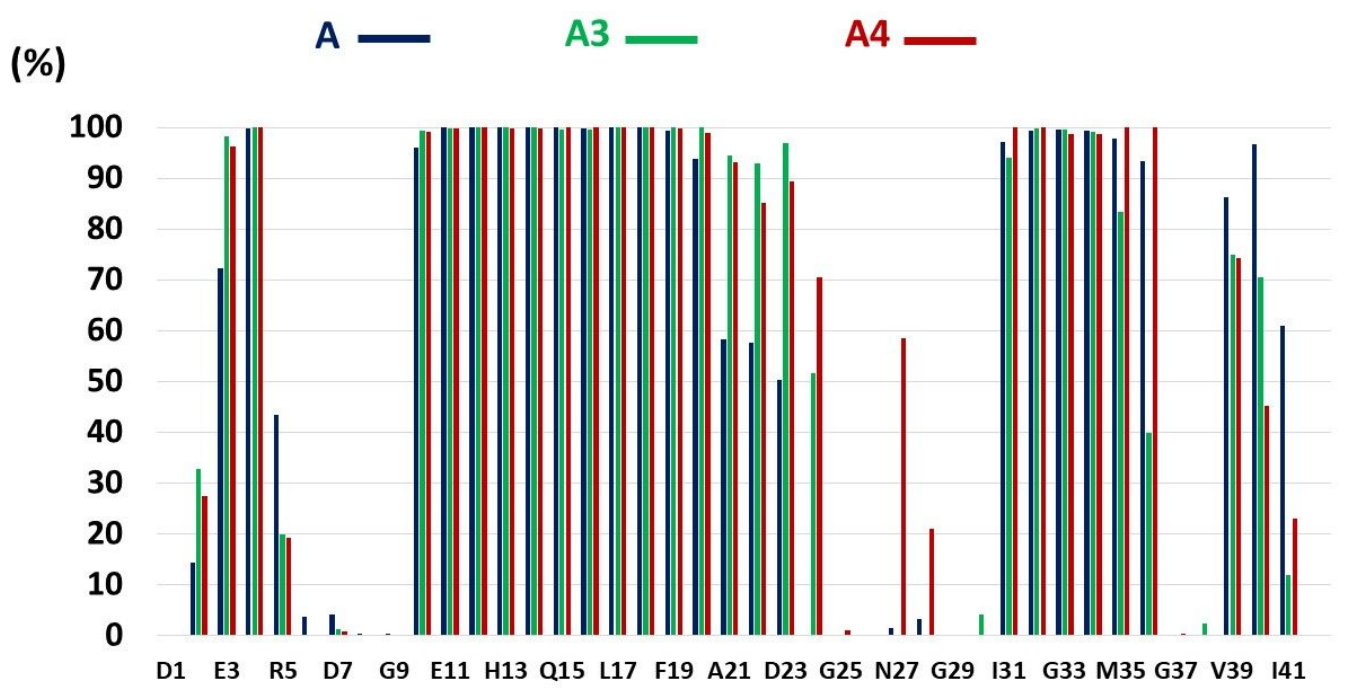

Figure S25: DSSP analysis for models A, A3 and A4 calculated from the last $5 \mathrm{~ns}$. 


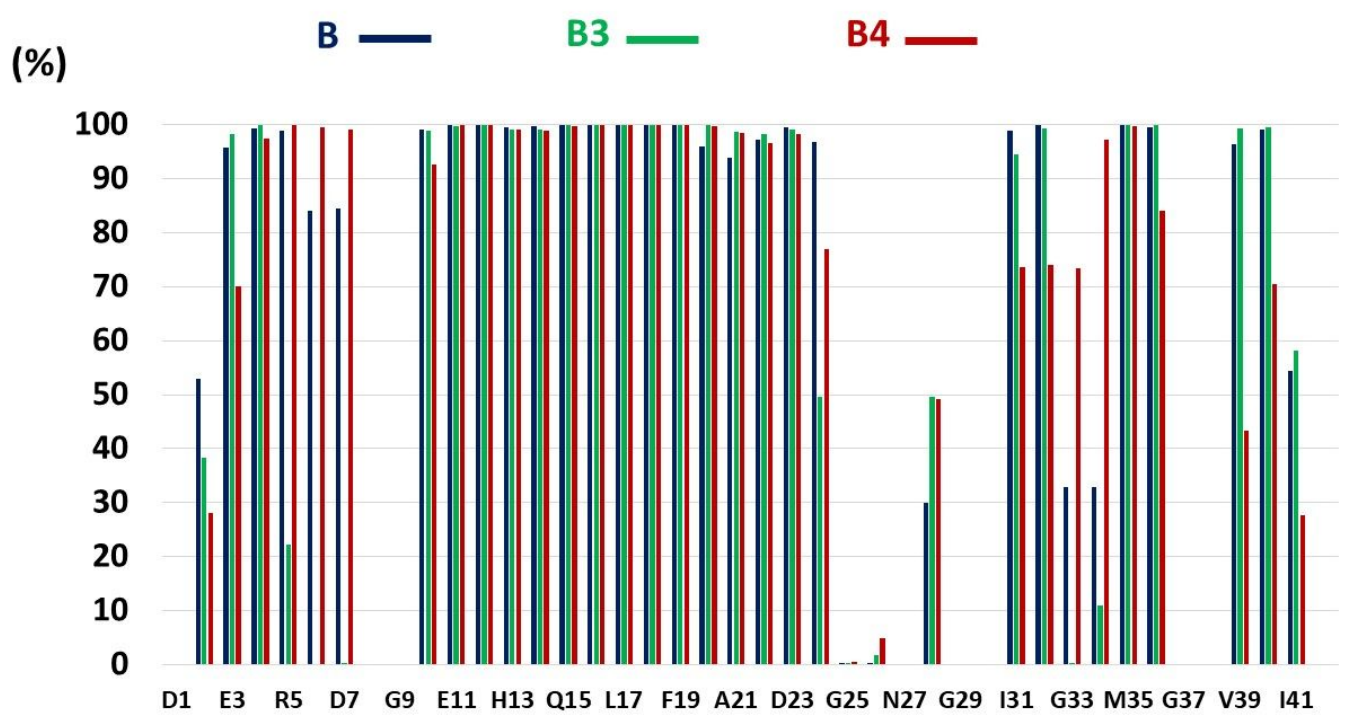

Figure S26: DSSP analysis for models B, B3 and B4 calculated from the last 5 ns. 


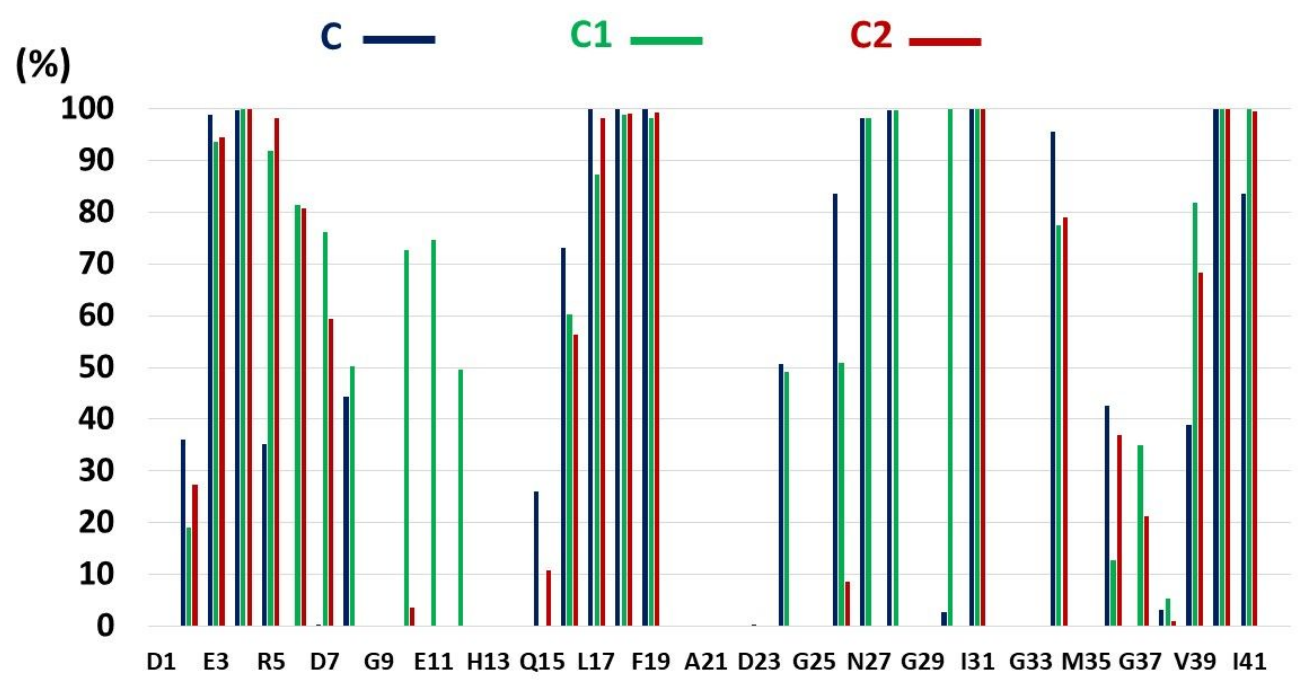

Figure S27: DSSP analysis for models C, C1 and C2 calculated from the last 5 ns. 
Table 1: A description of the location of residues along $\beta$-strands and U-turns domains of $\mathrm{A} \beta$ fibrils - models $\mathrm{A}, \mathrm{B}$ and $\mathrm{C}$ - originated from ssNMR structures.

\begin{tabular}{|c|c|c|}
\hline Model & $\beta$-strand domains & U-turn domains \\
\hline A & $10-22,30-40$ & $23-29$ \\
\hline B & $17-26,31-42$ & $27-30$ \\
\hline C & $2-6,15-18,26-28,30-32,39-42$ & $7-14,19-25,33-38$ \\
\hline
\end{tabular}




\section{References}

1. Luhrs, T.; Ritter, C.; Adrian, M.; Riek-Loher, D.; Bohrmann, B.; Dobeli, H.; Schubert, D.; Riek, R. (2005) 3D structure of Alzheimer's amyloid-beta(1-42) fibrils. Proc Natl Acad Sci USA 102, 17342-7.

2. Petkova, A. T.; Yau, W. M.; Tycko, R., (2006) Experimental constraints on quaternary structure in Alzheimer's beta-amyloid fibrils. Biochemistry 45, 498-512.

3. Walti, M. A.; Ravotti, F.; Arai, H.; Glabe, C. G.; Wall, J. S.; Bockmann, A.; Guntert, P.; Meier, B. H.; Riek, R. (2016) Atomic-resolution structure of a diseaserelevant Abeta(1-42) amyloid fibril. Proc Natl Acad Sci USA 113, E4976-84.

4. Whittingham, J. L.; Scott, D. J.; Chance, K.; Wilson, A.; Finch, J.; Brange, J.; Guy Dodson, G. (2002) Insulin at $\mathrm{pH} 2$ : structural analysis of the conditions promoting insulin fibre formation. $J$ Mol Biol 318, 479-90.

5. Turnell, W. G.; Finch, J. T. (1992) Binding of the dye congo red to the amyloid protein pig insulin reveals a novel homology amongst amyloid-forming peptide sequences. J Mol Biol 227, 1205-23.

6. Lee, M. S.; Feig, M.; Salsbury, F. R.; Brooks, C. L. (2003) New analytic approximation to the standard molecular volume definition and its application to generalized born calculations. J Comput Chem 24, 1348-1356.

7. Lee, M. S.; Salsbury, F. R.; Brooks, C. L. (2002) Novel generalized Born methods. J Chem Phys 116, 10606-10614.

8. Israelachvili, J.; Pashley, R. (1982) The hydrophobic interaction is long range, decaying exponentially with distance. Nature 300, 341-2.

9. Kumar, S.; Nussinov, R. (2002) Close-range electrostatic interactions in proteins. ChemBioChem 3, 604-17.

10. Burley, S. K.; Petsko, G. A. (1985) Aromatic-aromatic interaction: a mechanism of protein structure stabilization. Science 229, 23-8. 